\title{
A NOTE ON ASSORTATIVE MATING AND ARTIFICIAL SELECTION
}

\author{
M. SOLLER \\ Department of Genetics, Hebrew University, Jerusalem, Israel \\ and \\ A. GENIZI \\ Department of Statistics, Agricultural Research Organisation, Bet Dagon, Israel

\section{SUMMARY} \\ A simple method is presented for determining the effect on the genetic \\ variance of a quantitative trait, of selection followed by various degrees of \\ assortative mating.
}

Received 25.i.75

RECENTLY, Baker (1973) compared the effects of perfect assortative mating subsequent to truncation selection on the additive genotypic variance $(V)$ of the following generation, to the effects of truncation selection alone. The purpose of this note is to present a simple method of deriving a close approximation to these results. Our derivation also allows the effects of imperfect assortative mating to be readily obtained. We proceed as follows:

Let $h^{2}$ be the standardised additive genotypic variance found in a population. Then under Hardy-Weinberg equilibrium conditions, it is clear that $h^{2}$ will remain constant from generation to generation. That is, each generation an amount of additive genotypic variance equal to $h^{2}$ is produced. As is well known (see, e.g. Lush, 1945), under Hardy-Weinberg conditions two sources each contribute half of the total additive genotypic variance each generation. These are:

1. Mendelian segregation, which generates differences between the progeny of any particular parental pair, and

2. Random mating, which produces differences between the mean additive genotypic values of the various individual parental pairs.

For our purposes, differences between the mean additive genotypic values of individual parental pairs can also be considered as arising from two sources:

2.1. Differences in the expected additive genotypic values of parental pairs having different mean phenotypic values. The variation of mean phenotypic values of parental pairs is trivially equal to

$$
\frac{\lambda(1+r)}{2}
$$

where $\lambda=$ the proportion of the phenotypic variance remaining in the selected population after truncation. It has been shown (Pearson, 1903) that $\lambda$ is equal to

$$
1-\left(\frac{z_{p}\left(z_{p}-p x_{p}\right)}{p^{2}}\right)
$$


where $p$ is the proportion selected, $z_{p}$ and $x_{p}$ are the ordinate and abscissa, respectively, of the normal curve at the point of truncation. $r=$ the phenotypic correlation between mates in the selected group.

Thus, the variance of expected additive genotypic values between parental pairs, if judged solely by their mean phenotypic values, would be

$$
\frac{\left(h^{2}\right)^{2}}{2} \lambda(1+r) \text {. }
$$

2.2. Differences in the mean additive genotypic values of individual parental pairs all having the same mean phenotypic value. It has been shown (Soller, 1964) that (2.2) is equal to

$$
\frac{h^{2}\left(1-h^{2}\right)}{2} \text {. }
$$

Summing all contributions, we have for the additive genotypic variance of the generation following truncation selection and assortative mating

$$
\begin{aligned}
V & =(1)+(2.1)+(2.2) \\
& =\frac{h^{2}}{2}+\frac{\left(h^{2}\right)^{2}}{2} \lambda(1+r)+\frac{h^{2}\left(1-h^{2}\right)}{2} \\
& =h^{2}-\frac{\left(h^{2}\right)^{2}}{2}[1-\lambda(1+r)] .
\end{aligned}
$$

For assortative mating alone, we have

$$
V=h^{2}+\frac{\left(h^{2}\right)^{2}}{2} r
$$

and for truncation selection alone, we have

$$
V=h^{2}-\frac{\left(h^{2}\right)^{2}}{2}(1-\lambda) \text {. }
$$

Table 1 shows value of $\lambda$ for various proportions selected, and the calculated ratios of additive genotypic variance after selection and assortative

\begin{tabular}{|c|c|c|c|c|c|}
\hline \multirow{2}{*}{$\begin{array}{l}\text { Proportion } \\
\text { selected } \\
\text { (p) }\end{array}$} & \multirow{2}{*}{$\begin{array}{l}\text { Phenotypic } \\
\text { variance } \\
(\lambda)\end{array}$} & \multicolumn{4}{|c|}{$\begin{array}{c}\text { Ratio of additive genotypic variance: } \\
\text { Heritability }\end{array}$} \\
\hline & & 0.2 & 0.4 & $0 \cdot 6$ & $0 \cdot 8$ \\
\hline 0.50 & 0.364 & 1.039 & 1.083 & $1 \cdot 135$ & $1 \cdot 195$ \\
\hline 0.20 & 0.219 & 1.024 & 1.052 & $1 \cdot 086$ & $1 \cdot 127$ \\
\hline $0 \cdot 10$ & $0 \cdot 170$ & 1.019 & 1.041 & 1.068 & $1 \cdot 102$ \\
\hline 0.01 & $0 \cdot 108$ & $1 \cdot 012$ & 1.026 & $1 \cdot 044$ & 1.067 \\
\hline
\end{tabular}
mating to additive genotypic variance after selection and random mating. It should be noted that accurate values for $\lambda$ can be obtained only by interpolating to three additional places in table III (The Normal Probability

TABLE 1

Phenotypic variance after truncation selection, and ratio of additive genotypic variance after assortative mating to additive genotypic variance after random mating 
Integral) and Table II (Ordinates of the Normal Distribution) of Fisher and Yates (1963). When the values of table 1 are compared to those in the corresponding Table 4 of Baker (1973), it can be seen that the values obtained are either very close or identical to those obtained by Baker for the case of 100 loci with initial gene frequencies of $0 \cdot 5$. Baker's (1973) results also show that these ratios are relatively unaffected by the number of loci involved or by gene frequencies at the loci (except when heritability is high and selection intense). Thus the formulas presented here should be of general utility in approximating the short term of effects of selection and/or assortative mating on the additive genotypic variance of an offspring population.

\section{REFERENGES}

BAKER, R. J. 1973. Assortative mating and artificial selection. Heredity, 31, 231-238.

Fisher, R. A., AND YATES, F. 1963. Statistical Tables for Biological, Agricultural and Medical Research. Oliver and Boyd. Edinburgh.

Lush, J. L. 1945. Animal Breeding Plans, Third ed. Iowa State College Press, Ames, Iowa. PEARSON, K. 1903. On the influence of natural selection on the variability and correlation of organs. Phil. Trans. Roy. Soc. Lond., A200, 1-66.

SOLLER, M. 1964. Standard error of expected breeding values. 7. Dairy Sci., 47, 303-305. 